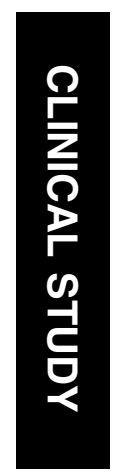

${ }^{1}$ Department of Ophthalmology, Oxford Eye Hospital, University of Oxford, Headley Way,

Oxford, UK

${ }^{2}$ Vienna Reading Center, Department of Ophthalmology, Medical University of Vienna, Waehringer Guertel, Vienna, Austria

${ }^{3}$ Austin Hospital, Heidelberg, Victoria, Australia

${ }^{4}$ Oxford University Clinical Academic Graduate School, University of Oxford Headley Way, Oxford, UK

${ }^{5}$ Nuffield Laboratory of Ophthalmology, University of Oxford, Headley Way, Oxford, UK

${ }^{6}$ Department of Ophthalmology, University of Bonn, Bonn, Germany

Correspondence: NV Chong, Department of Ophthalmology, Oxford Eye Hospital, University of Oxford, Headley Way, Headington, Oxford OX3 9DU, UK

Tel: + 44 (0)1865 234736;

Fax: + 44 (0)1865 234515 .

E-mail: victor@eretina.org

Received: 22 December 2011

Accepted in revised form: 15 April 2012

Published online: 15 June 2012

\section{Two-wavelength fundus autofluorescence and macular pigment optical density imaging in diabetic macular oedema}

SM Waldstein ${ }^{1,2}$, D Hickey ${ }^{1,3}$, I Mahmud ${ }^{4}$, CA Kiire ${ }^{1}$, P Charbel Issa ${ }^{5,6}$ and NV Chong ${ }^{1}$

\begin{abstract}
Purpose To evaluate the application of 488 and $514 \mathrm{~nm}$ fundus autofluorescence (FAF) and macular pigment optical density (MPOD) imaging in diabetic macular oedema (DMO) and to demonstrate the typical imaging features.

$514 \mathrm{~nm}$ FAF, provides a valuable addition to OCT in the evaluation of DMO and is clinically useful in rapid en-face assessment of the central macula.

Eye (2012) 26, 1078-1085; doi:10.1038/eye.2012.100; published online 15 June 2012
\end{abstract}

Patients and Methods A hundred and twentyfive eyes of 71 consecutive patients with diabetic retinopathy who underwent examination at a specialist university clinic employing a modified Heidelberg Retina Angiograph, using two different light sources of 488 and $514 \mathrm{~nm}$ wavelength, were retrospectively reviewed. MPOD images were calculated using modified Heidelberg Eye Explorer software. All images were evaluated by two independent masked graders. Features from FAF and MPOD images were correlated with optical coherence tomography (OCT) imaging findings and inter-grader variability, sensitivity and specificity were calculated using OCT as reference.

Results Sixty-seven eyes had DMO on OCT. The inter-grader variability was 0.84 for $488 \mathrm{~nm}$ FAF, 0.63 for $514 \mathrm{~nm}$ FAF and 0.79 for MPOD imaging. Sensitivity and specificity for detection of DMO were 80.6 and $89.7 \%$ for $\mathbf{4 8 8} \mathrm{nm}$ FAF; 55.2 and $\mathbf{9 4 . 8 \%}$ for $\mathbf{5 1 4} \mathrm{nm}$ FAF; and 80.6 and $91.4 \%$ for MPOD imaging. In $488 \mathrm{~nm}$ FAF and MPOD imaging, DMO was better visualised in comparison with 514 nm FAF imaging, $P<0.01$. MPOD revealed displacement of macular pigment by intraretinal cysts.

Conclusion MPOD imaging, and

particularly its combination with $488 \mathrm{~nm}$ and
Keywords: diabetic macular oedema; fundus autofluorescence; macular pigment

\section{Introduction}

Diabetic macular oedema (DMO) is a major cause of the visual impairment in patients with diabetes mellitus. ${ }^{1}$ Focal or grid laser photocoagulation has long been the only treatment for this condition. However, recent pharmacological developments have added new promising treatment options, including the intravitreal application of steroids $\mathrm{s}^{2,3}$ and inhibitors of vascular endothelial growth factor. ${ }^{4,5}$ Such therapeutic progress has resulted in an increasing demand for objective methods to identify, monitor and classify DMO. The availability of various treatment options may require stratification of disease subtypes that may respond differently to specific treatment approaches.

Stereo fundus photography, which requires specialist viewing equipment, and fluorescein angiography (FA), which is invasive, have long been the gold standard for assessing and documenting DMO. Recently, retinal thickness measurement ${ }^{6-8}$ and structural assessment ${ }^{9,10}$ by non-invasive optical coherence tomography (OCT) have increasingly been used. However, the functional correlation with retinal thickness 
measurements alone is poor, 11,12 and cross-sectional structural assessment may miss alterations outside the scan lines that would be detected by topographic imaging. ${ }^{13}$

Fundus autofluorescence (FAF) imaging using a confocal scanning laser ophthalmoscope (cSLO) allows for topographic assessment of macular pathology with high resolution. Pece et $a l^{14}$ found that conventional FAF imaging with a wavelength of $488 \mathrm{~nm}$ provides a sensitive, rapid and non-invasive way of assessing DMO, correlating well with FA and findings on OCT imaging. Vujosevic and colleagues ${ }^{15}$ also described good correlation between FAF, OCT, FA and central field microperimetry. FAF imaging showed $81 \%$ sensitivity and $69 \%$ specificity in detecting cystic macular oedema of non-diabetic aetiology in a study by McBain and colleagues. ${ }^{16}$

Most commercially available cSLOs for FAF imaging employ blue laser light with a wavelength of $488 \mathrm{~nm}$ for excitation. ${ }^{17,18}$ The macular pigment (MP), however, with its high concentration of lutein and zeaxanthin, absorbs blue light to a large extent and thus greatly attenuates the FAF signal from the central macula. ${ }^{19,20}$ Given that the underlying pathological mechanisms in DMO could potentially influence MP distribution, ${ }^{16}$ the interpretation of conventional $488 \mathrm{~nm}$ FAF images in patients with DMO may be difficult.

Excitation light at slightly longer wavelengths, for example, using a green laser centred at $514 \mathrm{~nm}$, is much less absorbed by MP and hence might offer advantages in such clinical situations. The sequential use of both 488 and $514 \mathrm{~nm}$ FAF allows for the calculation of MP optical density (MPOD) maps that topographically illustrate the relative distribution of $\mathrm{MP}^{21-23}$ A quantitative comparison of MPOD in diabetic and non-diabetic patients has recently been performed by Lima and co-workers. ${ }^{24}$

We hypothesised that qualitative evaluation of $514 \mathrm{~nm}$ FAF and MPOD could provide additional useful information in the assessment of DMO. Thus, the purpose of this study was (1) to compare the sensitivity and specificity of two-wavelength FAF and MPOD imaging for the detection of DMO in patients with diabetic retinopathy and (2) to systematically describe characteristic FAF and MPOD appearances in DMO and consider the possible causes for these.

\section{Patients and methods}

This research adhered to the tenets of the Declaration of Helsinki and approval was granted by the Oxfordshire A Research Ethics Committee, Oxford, UK.

In a retrospective cross-sectional study, a chart review of all patients with diabetic retinopathy who underwent OCT and two-wavelength FAF imaging in the diabetic retinopathy clinic of a university hospital between May 2009 and November 2010 was conducted. One hundred and twenty-five eyes of 71 patients were included.

Inclusion criteria were as follows: (1) the presence of diabetic retinopathy with or without DMO, (2) clear ocular media that allow recording of high-quality FAF images and (3) availability of both two-wavelength FAF and OCT imaging within a 2-week period. Exclusion criteria were the presence of any ocular comorbidity affecting the macula, such as retinal vein occlusion or age-related macular degeneration.

\section{Evaluation and image acquisition}

FAF and MPOD imaging were performed as has been previously described. ${ }^{21-23}$ Briefly, a cSLO (modified HRA classic, Heidelberg Engineering, Heidelberg, Germany) with an external source of both a solid-state laser emitting at $488 \mathrm{~nm}$ and an argon-ion laser emitting at $514 \mathrm{~nm}$ was employed. At a $30^{\circ}$ field of view, a series of 16 images was recorded at both 488 and $514 \mathrm{~nm}$ excitation. Modified Heidelberg Eye Explorer software was used for image processing. Mean images were calculated for both wavelengths separately and subtraction of the two averaged images was used to create an image representing the topographic MPOD distribution.

Additionally, all patients underwent a complete ophthalmological examination, including best corrected Snellen visual acuity testing and slit-lamp biomicroscopy. OCT, which we consider the clinical standard for the non-invasive diagnosis of DMO, was used as the reference investigation. ${ }^{6-8}$ For this we used a commercially available spectral domain (SD)-OCT instrument (Spectralis OCT, Heidelberg Engineering, software version 1.6.4.0). Each B-scan consisted of 512 A-scans and was averaged nine times using the ART mode. A $20^{\circ} \times 20^{\circ}$ scan pattern using 25 sections with an inter-scan distance of $240 \mu \mathrm{m}$ was recorded.

\section{Image grading and statistics}

All FAF and MPOD images were initially evaluated for the presence or absence of DMO by two independent graders (SMW, CAK). The graders were blinded to the patient, but could not be blinded to the mode of imaging because each of the employed technologies has unique image characteristics. The following scoring system was used in order to compare the visibility of DMO between the three technologies: (0) no DMO visible; (1) DMO suspected; and (2) DMO clearly visible, allowing a diagnosis to be made. The inter-grader agreement was calculated using Cohen's kappa. Before correlating the FAF and MPOD results with OCT findings, any 
discrepancies in grading were discussed until a consensus opinion was reached with a senior grader (PCI). A reading-centre certified grader (SMW) assessed OCT scans for signs of DMO, that is, intraretinal cysts, subfoveal neurosensory detachment and retinal thickening. Sensitivity and specificity of FAF and MPOD imaging methods were calculated using OCT as a reference as follows: Sensitivity $=$ true positives / (true positives + false negatives); specificity $=$ true negatives/ (true negatives + false positives). A score of ' 0 ' was regarded as 'negative' and scores of ' 1 ' or ' 2 ' were regarded as 'positive' in this analysis. The visibility of $\mathrm{DMO}$ according to the scoring system was compared between the imaging modalities for eyes with DMO on OCT using the Wilcoxon signed-rank test. All statistical tests were performed using PASW Statistics version 18 software (SPSS Inc., Chicago, IL, USA). The nominal significance level was set at 0.05 .

\section{Grey level analysis}

On a subset of images, linear grey value plots were performed with ImageJ software (version $1.43 \mathrm{u}$, National Institutes of Health, Bethesda, MD, USA) to illustrate the relative distribution of autofluorescence and MP abnormalities. Areas of 400 pixels length and 5 pixels height, centred through the region of interest, were analysed.

\section{Results}

\section{Demographic data}

Of the 125 eyes of 71 patients included, 67 eyes had signs of DMO and 58 showed no features of DMO on SD-OCT. The mean patient age was 63 years \pm 15 years (SD); 46 participants were male and 25 were female. All had signs of diabetic retinopathy on clinical examination.
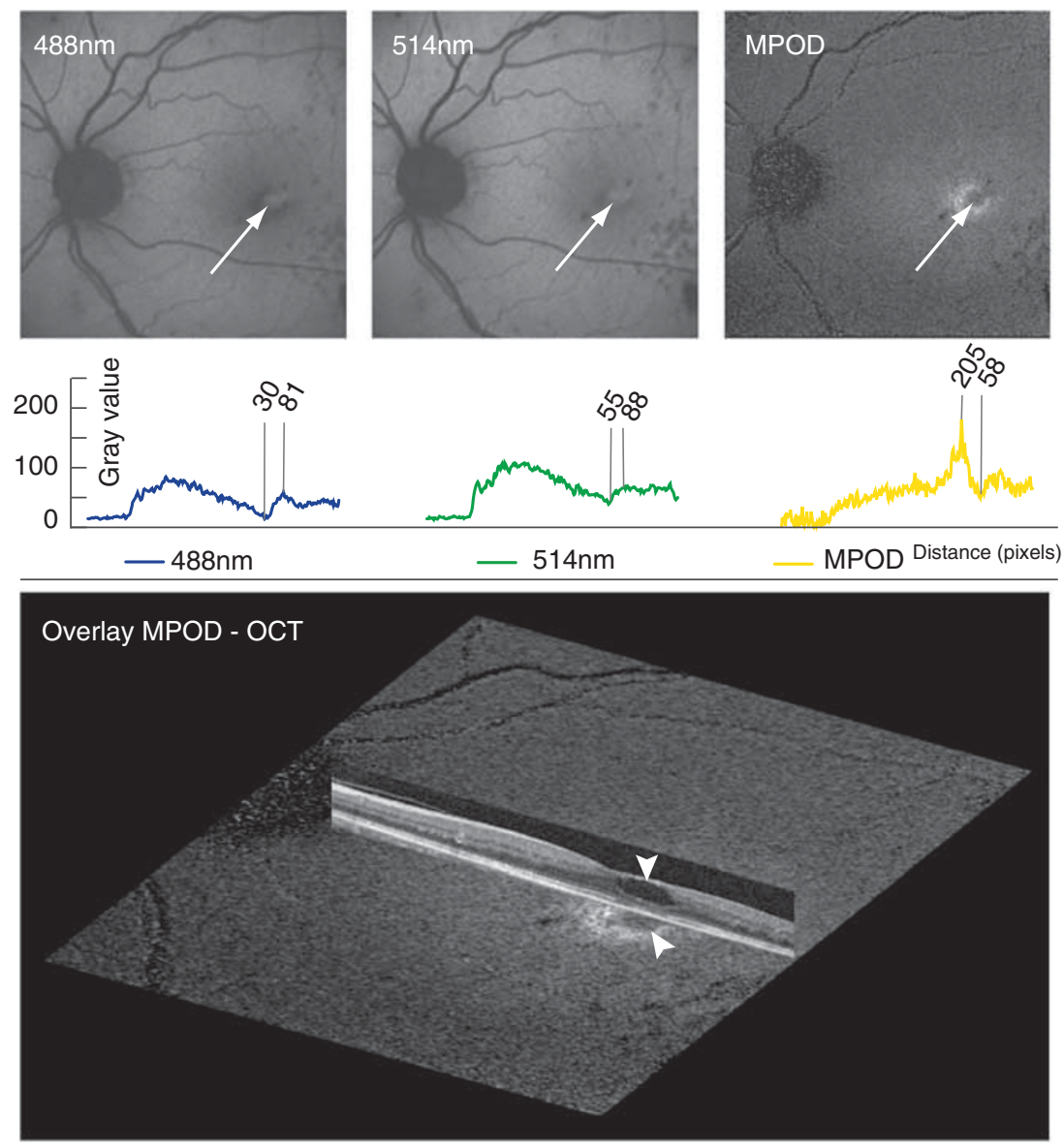

Figure 1 DMO on two-wavelength FAF and MPOD imaging. Cystic changes (white arrows) typically stand out due to displacement of MP. (Top left) The lateral displacement of MP within the cystic area results in reduced $488 \mathrm{~nm}$ FAF attenuation. The concentrated MP surrounding the cysts increases the contrast (49 grey levels difference) and allows a clear delineation of the lesion. (Top centre) The contrast is less pronounced using $514 \mathrm{~nm}$ light for FAF excitation (33 grey levels difference), which is less absorbed by MP and less effective in exciting fundus fluorophores. (Top right) MPOD imaging has even greater contrast (147 grey levels difference) than $488 \mathrm{~nm}$ FAF and confirms displacement of MP. (Bottom) The ovoid area of low MP density corresponds with a large intraretinal cyst on OCT (arrowheads). 


\section{Characteristic imaging findings}

On FAF images, DMO typically stands out as relatively bright, single or multiple, round or oval areas that are mostly bordered by darker rims. In all cases, the ovoid bright spots on FAF imaging correlated well with intraretinal cysts on OCT imaging. Figure 1 provides an example image with OCT correlation.

Grey value analysis (Figure 2) shows that the FAF signal intensity at the ovoid lesions is similar to the more eccentric background signal where MP concentration is very low, while the dark rims on FAF images clearly correspond to areas with high MP content on MPOD imaging (Figure 1). This suggests that cysts laterally displace MP, resulting in a loss of the normal MP-associated AF masking. The rims of attenuated signal with FAF imaging are caused by increased absorption of excitation light within the displaced, focally concentrated MP.

Imaging using $488 \mathrm{~nm}$ FAF alone cannot clearly distinguish between potential causes of focal hypofluorescence, which could be attributed to MP concentration as a result of the displacement by intraretinal cysts, other pathology such as haemorrhage or exudates or reduced tissue viability. Two-wavelength FAF imaging with MPOD mapping more effectively makes this distinction, offering advantages in the evaluation of focal hypofluorescence, as illustrated in Figure 3.

\section{Inter-observer agreement, sensitivity and specificity of FAF and MPOD imaging}

Cohen's kappa inter-observer agreement for the different imaging techniques was 0.84 for $488 \mathrm{~nm}$ FAF, 0.63 for $514 \mathrm{~nm}$ FAF and 0.79 for MPOD imaging. Sensitivity and specificity were, respectively, $80.6 \%$ and $89.7 \%$ for $488 \mathrm{~nm}$ FAF; $55.2 \%$ and $94.8 \%$ for $514 \mathrm{~nm}$ FAF; and $80.6 \%$ and $91.4 \%$ for MPOD imaging.

By categorising the appearance of DMO by the different imaging modalities, differences in efficacy were revealed according to the scoring system, as shown in Table 1. Of note, both $488 \mathrm{~nm}$ FAF and MPOD imaging were significantly more efficient in the diagnosis of DMO than 514 nm FAF, $P<0.01$ (Wilcoxon signed-rank test).
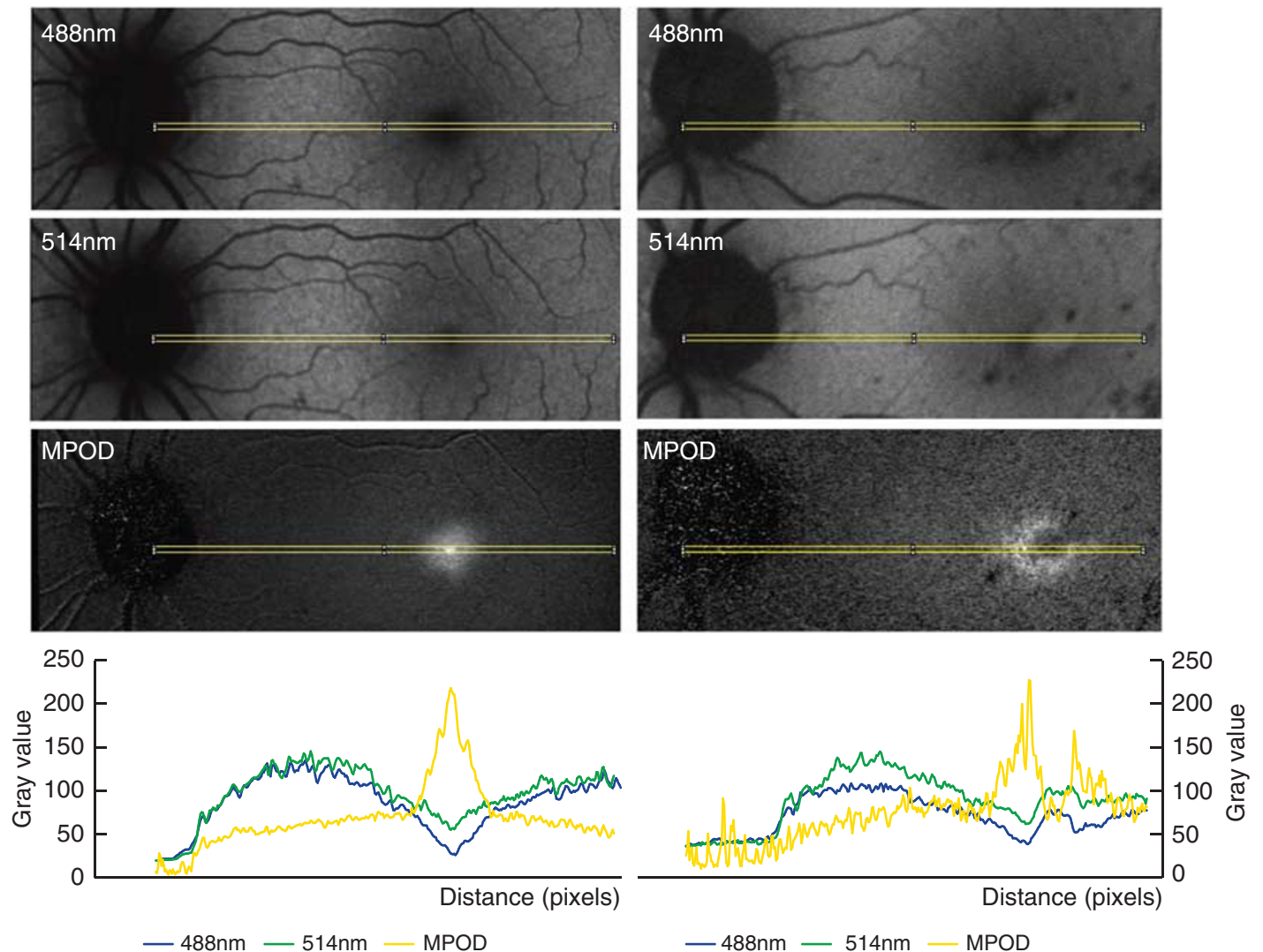

Figure 2 Grey value analysis. (Left column) In an eye without DMO, the concentration of MP shows a sharp, single peak in the grey value plot (graph, yellow line). (Right column) In an eye with DMO, the distribution of MP is disrupted by a cystic lesion (MPOD image; graph, yellow line). The 488 and $514 \mathrm{~nm}$ autofluorescence values (graph, blue and green lines) in the area of the cyst are comparable to normal background signal. Yellow bars identify the measurement area. 

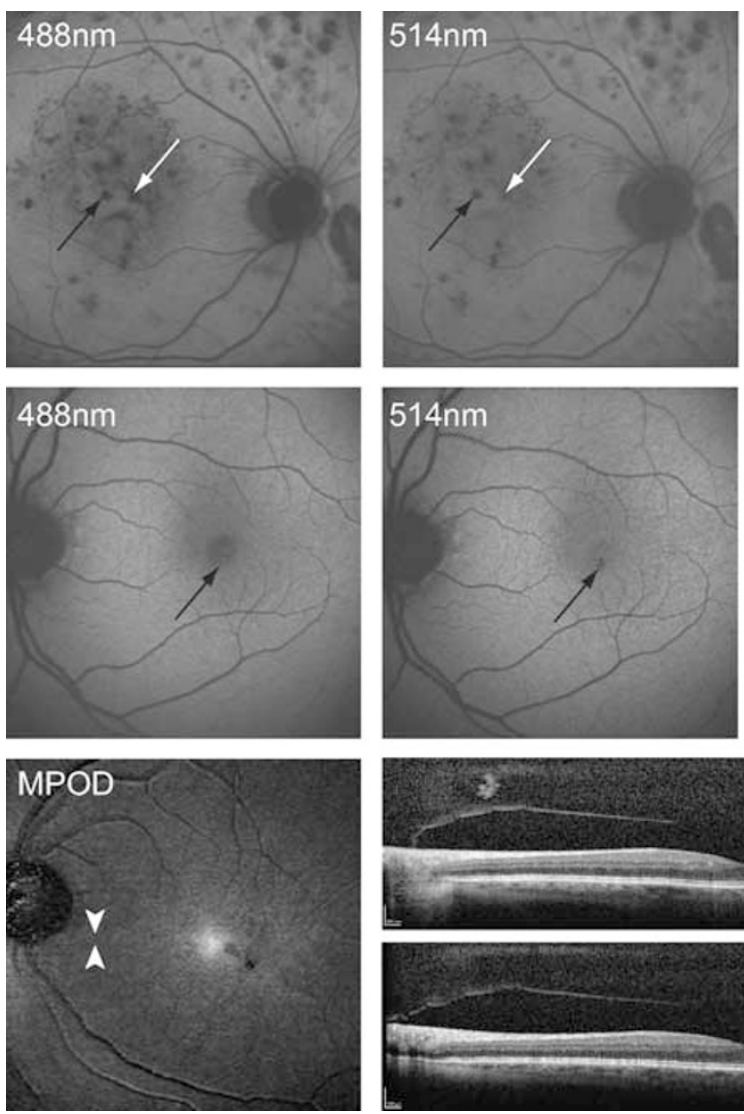
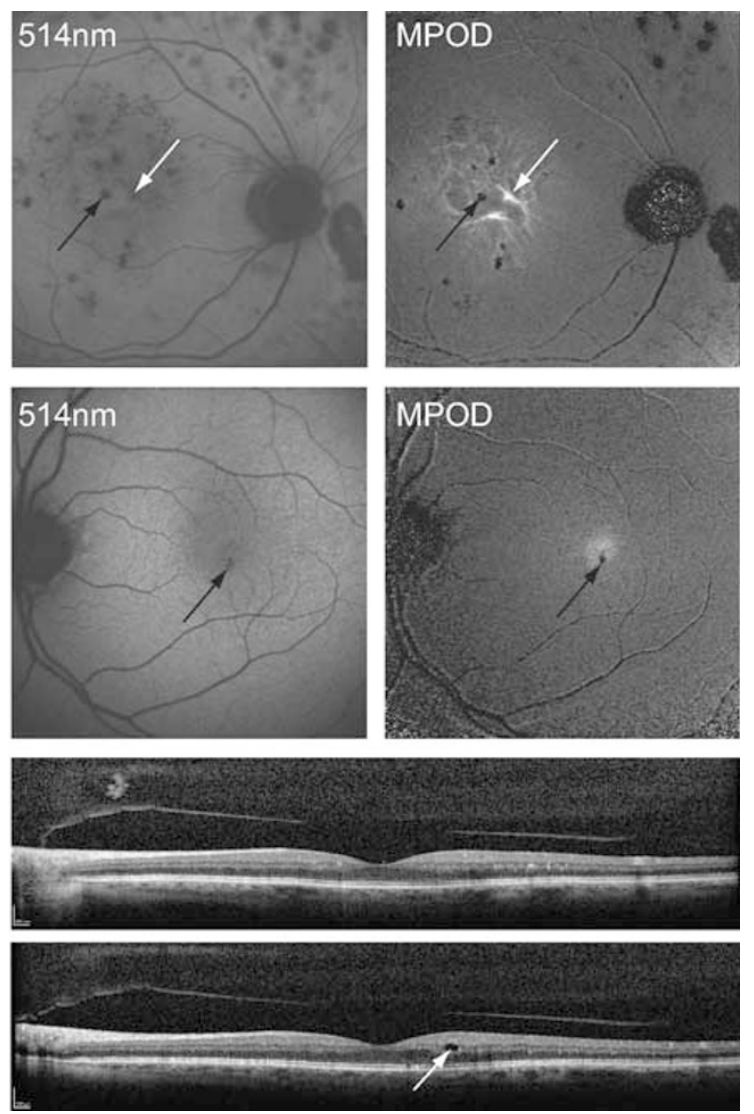

Figure 3 Evaluation of hypofluorescent lesions on FAF images. (Top left and centre) The distinction between focal accumulation of MP due to displacement by intraretinal cysts (white arrows), and haemorrhage (black arrows) is challenging in this example of conventional 488 and $514 \mathrm{~nm}$ FAF images. (Top right) MPOD imaging shows a striking contrast between MP accumulation and haemorrhage, as haemorrhages remain black, whereas accumulations of MP appear white. Overall, MPOD mapping gives comprehensive full-field information on tissue viability. (Middle left) The small foveal haemorrhage can easily be missed on $488 \mathrm{~nm}$ FAF because haemorrhage is masked by MP. (Middle centre and right) By contrast, 514FAF and even more MPOD imaging provide a clear depiction of the haemorrhage. Advantages of topographic en-face imaging. (Bottom left) In this example, a juxtafoveal cyst in its entire extension is clearly visible on the MPOD image as a dark ovoid spot. (Bottom right) On the central OCT scan, however, the cyst is not visible and could be overlooked if, for example, only one horizontal and one vertical scan were evaluated. A tightly spaced raster scan permitted detection of the cyst in the outer plexiform layer of the retina on another B-scan (arrow). Arrowheads indicate the OCT B-scan section planes.

We identified three distinct patterns of DMO on OCT, all of which were cystic: (1) predominantly foveal intraretinal cysts, 51 eyes $(76 \%)$; (2) predominantly extrafoveal intraretinal cysts, 5 eyes (7\%); and (3) diffuse, small intraretinal cysts, 11 eyes (16\%). 'Foveal' on OCT was defined as within a circular area of $1 \mathrm{~mm}$ diameter centred on the foveola. The sensitivities of all modes of FAF and MPOD imaging was highest for eyes with predominantly foveal intraretinal cysts on OCT imaging (90\%, 70\%, and $96 \%$ for $488 \mathrm{~nm}$ FAF, $514 \mathrm{~nm}$ FAF and MPOD, respectively). An overview of sensitivities for all OCT patterns and overall specificity is given in Table 2.

\section{Discussion}

This study was designed to compare the efficacy of $488 \mathrm{~nm}$ FAF, $514 \mathrm{~nm}$ FAF and MPOD imaging in DMO.
Using SD-OCT as the diagnostic standard, our results show that $488 \mathrm{~nm}$ FAF and MPOD imaging are very effective at detecting DMO. Both methods showed a high sensitivity of over $80 \%$. MPOD imaging was, however, the most specific. It was the easiest for the graders to evaluate because it provided the best contrast (Figure 2, grey level analysis). As visualisation of displaced MP is required for the indirect delineation of cysts, $514 \mathrm{~nm}$ FAF alone (which is not as strongly influenced by MP) was less reliable in the detection of DMO, as reflected by a rather low sensitivity of $55 \%$. Only in cases of extensive MP accumulation did signs of DMO become evident on $514 \mathrm{~nm}$ FAF.

Regarding morphological analysis, MPOD imaging in conjunction with FAF can provide better information on the aetiology of autofluorescence abnormalities than FAF imaging alone. For instance, hypofluorescent lesions on 
Table 1 Visibility of macular oedema on $488 \mathrm{~nm}$ FAF, $514 \mathrm{~nm}$ FAF and MPOD

\begin{tabular}{lccc}
\hline & $488 n m$ FAF & $514 n m$ FAF & MPOD \\
\hline Mean score $^{\mathrm{a}} \pm$ SD; & $1.43 \pm 0.8 ; 2$ & $0.93 \pm 0.9 ; 1$ & $1.49 \pm 0.8 ; 2$ \\
Median score $_{P \text {-value }}$ & $<0.01$ & $<0.01$ \\
\hline
\end{tabular}

Abbreviations: DMO, diabetic macular oedema; FAF, fundus autofluorescence; MPOD, macular pigment optical density; OCT, optical coherence tomography.

Average scores for eyes with DMO on OCT are shown.

a Possible scoring values: (0) no DMO visible; (1) DMO suspected; (2) DMO clearly visible.

${ }^{\mathrm{b}}$ Wilcoxon signed-rank test.

Table 2 Inter-observer variability, sensitivity and specificity overview

\begin{tabular}{llllll}
\hline & & \multicolumn{4}{c}{ Sensitivity $^{\mathrm{a}}$} \\
\cline { 3 - 5 } & Kappa & $\begin{array}{c}\text { Overall } \\
(\%)\end{array}$ & $\begin{array}{c}\text { Foveal } \\
\text { cysts } \\
(\%)\end{array}$ & $\begin{array}{c}\text { Extrafoveal } \\
\text { or diffuse } \\
\text { cysts (\%) }\end{array}$ & $\begin{array}{c}\text { Specificity } \\
\text { Overall } \\
(\%)\end{array}$ \\
\hline $488 \mathrm{~nm}$ FAF & 0.84 & 80.6 & 90.0 & 60.8 & 89.7 \\
$514 \mathrm{~nm}$ FAF & 0.63 & 55.2 & 70.0 & 20.0 & 94.8 \\
MPOD & 0.79 & 80.6 & 96.0 & 45.5 & 91.4 \\
\hline
\end{tabular}

Abbreviations: FAF, fundus autofluorescence; MPOD, macular pigment optical density; OCT, optical coherence tomography.

a Predominately foveal, extrafoveal or diffuse pattern of DMO, as seen on OCT.

FAF, caused by focal accumulation of displaced MP, can be indistinguishable from other causes of hypofluorescence (eg, reduced tissue viability or blocking by exudates or haemorrhage). In contrast, MPOD imaging can selectively depict the MP and differentiate between these pathologies (Figure 3).

In comparison with OCT imaging, an advantage of FAF and MPOD examination lies in their topographic en-face imaging properties. Although current SD-OCT instruments with raster scanning modes are much less problematic than the older time-domain OCT instruments (which is often only scanned once horizontally and once vertically), the examiner could overlook small cystic changes because of wide raster spacing. In FAF or MPOD imaging, the entire macula can be comprehensively assessed with a $30^{\circ}$ field of view in a single rapid recording (Figure 3).

A limitation of FAF and MPOD technologies lies in the detection of extrafoveal and diffuse DMO, as reflected by a low average sensitivity of $42 \%$ for these morphologic types of DMO in our study. The lack of specific FAF findings in extrafoveal disease can be attributed to the low MP concentration eccentric to the fovea, and hence the absence of its contrast enhancing properties that are necessary for the delineation of cystic changes.
MPOD and FAF alterations in DMO may be of functional and pathophysiological significance. Our data show that autofluorescence per se is minimally altered by the presence of intraretinal cysts. Instead, the data suggest that the changes seen on FAF are more likely to be due to the altered distribution of MP resulting from the cysts. The autofluorescence signal from the centre of the intraretinal cysts is similar to that of the peripheral retina, where autofluorescence attenuation by MP is not relevant because of the absence of MP in this area. The cysts displace MP, resulting in its focal accumulation around the cysts. Thus the altered distribution of MP is likely to be the reason for the (indirect) visibility of DMO on FAF imaging. Previous studies combining FAF with microperimetry, nevertheless, showed that, in DMO, abnormalities on FAF imaging correlate with reduced visual function. ${ }^{15}$ It is possible that the altered MP distribution contributes to functional loss, as it has been shown that MP increases macular function and reduces glare. ${ }^{25-27}$ Moreover, focal displacement of MP and the resulting reduced protection from blue-light exposure might result in increased photo-oxidative stress in the outer retina. This could potentially lead to increased light-associated retinal damage, as suggested for another disease, macular telangiectasia type 2 , with its characteristic central loss of MP. ${ }^{23,28}$

Apart from focal displacement of MP as demonstrated in our study, patients with diabetic maculopathy have also been shown to have a reduction in MP levels, which might be caused by increased oxidative stress. ${ }^{29}$ As MPs seem to have an important role in the homoeostasis of retinal oxidative load, lack of MP in the context of incipient oxidative damage in diabetes mellitus may actually contribute substantially to the pathogenesis of DMO. Whether focal or global depletion of MP increases the risk of further oxidative damage, and therefore progression of $\mathrm{DMO}$, is yet to be determined. In this context, further longitudinal investigations in patients with $\mathrm{DMO}$, focusing on disease duration and treatment response, are needed to correlate alterations in the MP density and the distribution with visual acuity and other tests of retinal function.

This study has several limitations. Firstly, our sample was not specifically stratified according to the severity of cataract. As was recently demonstrated by Sasamoto et $a l^{30}$ cataract can lead to decreased visualisation of MP in two-wavelength FAF imaging. However, we excluded patients with optical media opacities that would not allow for FAF imaging and we did not perform quantitative analysis of the MPOD. Secondly, the retrospective nature of the study could have introduced a selection bias that we could not balance for. That is, patients with severe DMO might have been scheduled for FAF examination less often. Nevertheless, in our chart 
review we were able to identify a large number of patients with DMO with a wide range of disease severity. Thirdly, our study was cross-sectional and did not feature longitudinal data. A long-term assessment of patients with diabetes would be needed in the future to elucidate whether, for example, the distribution of MP returns to normal after successful treatment of DMO.

\section{Conclusion}

In conclusion, $488 \mathrm{~nm}$ FAF and MPOD imaging are both sensitive and specific methods for rapid, non-invasive and en-face topographic detection of DMO. MPOD mapping using two-wavelength FAF is of substantial value in differentiating between various causes of focal hypofluorescence, and shows the displacement of MP by intraretinal cysts. The combined use of $488 \mathrm{~nm}, 514 \mathrm{~nm}$ and MPOD imaging may provide a detailed and clinically useful multi-modal assessment tool for patients with DMO.

\section{Summary}

What was known before

- DMO can be detected by conventional blue-light fundus autofluorescence imaging and typically shows foveal lesions with increased autofluorescence.

- The ability of novel green light fundus autofluorescence and macular pigment imaging to detect macular oedema was unclear.

What this study adds

- Conventional blue-light fundus autofluorescence and macular pigment imaging are more sensitive in the detection of macular oedema compared with green light fundus autofluorescence.

- Macular pigment imaging indicates that DMO-associated foveal lesions of increased autofluorescence on FAF images are caused by the displacement of macular pigment.

- Topographic macular pigment imaging may detect subtle cystic changes that could be missed on optical coherence tomography.

\section{Conflict of interest}

The authors declare no conflict of interest.

\section{Acknowledgements}

PCI was supported by a Marie Curie Intra-European Fellowship (no.: 237238; the Seventh European Community Framework Program, European Commission, Brussels, Belgium). DH was supported by a Worshipful Company of Barbers-Waitangi Foundation Fellowship and The University of Auckland. The sponsors or funding organisations had no role in the design or the conduct of this research. The contents of this publication reflect only the author's views and not the views of the funding organisations.

\section{References}

1 Moss SE, Klein R, Klein BEK. The 14-year incidence of visual loss in a diabetic population. Ophthalmology 1998; 105(6): 998-1003.

2 Diabetic Retinopathy Clinical Research Network. A randomized trial comparing intravitreal triamcinolone acetonide and focal/grid photocoagulation for diabetic macular edema. Ophthalmology 2008; 115(115): 1447-1459.

3 Diabetic Retinopathy Clinical Research Network. Threeyear follow-up of a randomized trial comparing focal/grid photocoagulation and intravitreal triamcinolone for diabetic macular edema. Arch Ophthalmol 2009; 127(3): 245-251.

4 Elman MJ, Aiello LP, Beck RW, Bressler NM, Bressler SB, Edwards AR et al. Randomized trial evaluating ranibizumab plus prompt or deferred laser or triamcinolone plus prompt laser for diabetic macular edema. Ophthalmology 2010; 117(6): 1064-1077.

5 Mitchell P, Bandello F, Schmidt-Erfurth U, Lang GE, Massin P, Schlingemann RO et al. The RESTORE Study: ranibizumab monotherapy or combined with laser versus laser monotherapy for diabetic macular edema. Ophthalmology 2011; 118(4): 615-625.

6 Strom C, Sander B, Larsen N, Larsen M, Lund-Andersen H. Diabetic macular edema assessed with optical coherence tomography and stereo fundus photography. Invest Ophthalmol Vis Sci 2002; 43(1): 241-245.

7 Browning DJ, McOwen MD, Bowen RM Jr, O'Marah TL. Comparison of the clinical diagnosis of diabetic macular edema with diagnosis by optical coherence tomography. Ophthalmology 2004; 111(4): 712-5.

8 Virgili G, Menchini F, Dimastrogiovanni AF, Rapizzi E, Menchini U, Bandello F et al. Optical coherence tomography versus stereoscopic fundus photography or biomicroscopy for diagnosing diabetic macular edema: a systematic review. Invest Ophthalmol Vis Sci 2007; 48(11): 4963-4973.

9 Otani T, Kishi S, Maruyama Y. Patterns of diabetic macular edema with optical coherence tomography. Am J of Ophthalmol 1999; 127(6): 688-693.

10 Kang SW, Park CY, Ham D-I. The correlation between fluorescein angiographic and optical coherence tomographic features in clinically significant diabetic macular edema. Am J Ophthalmol 2004; 137(2): 313-322.

11 Goebel W, Kretzchmar-Gross T. Retinal thickness in diabetic retinopathy: a study using optical coherence tomography (OCT). Retina 2002; 22(6): 759-767.

12 Alasil T, Keane PA, Updike JF, Dustin L, Ouyang Y, Walsh AC et al. Relationship between optical coherence tomography retinal parameters and visual acuity in diabetic macular edema. Ophthalmology 2010; 117(12): 2379-2386.

13 Helb HM, Charbel Issa P, Fleckenstein M, Schmitz-Valckenberg S, Scholl HP, Meyer $\mathrm{CH}$ et al. Clinical evaluation of simultaneous confocal scanning laser ophthalmoscopy imaging combined with high-resolution, spectral-domain optical coherence tomography. Acta Ophthalmol 2010; 88(8): 842-849.

14 Pece A, Isola V, Holz F, Milani P, Brancato R. Autofluorescence imaging of cystoid macular edema in diabetic retinopathy. Ophthalmologica 2010; 224(4): 230-235. 
15 Vujosevic S, Casciano M, Pilotto E, Boccassini B, Varano M, Midena $\mathrm{E}$ et al. Diabetic macular edema: fundus autofluorescence and functional correlations. Invest Ophthalmol Vis Sci 2011; 52(1): 442-448.

16 McBain VA, Forrester JV, Lois N. Fundus autofluorescence in the diagnosis of cystoid macular oedema. Br J Ophthalmol 2008; 92(7): 946-949.

17 von Ruckmann A, Fitzke FW, Bird AC. Distribution of fundus autofluorescence with a scanning laser ophthalmoscope. Br J Ophthalmol 1995; 79(5): 407-412.

18 Bellmann C, Holz FG, Schapp O, Völcker HE, Otto TP. [Topography of fundus autofluorescence with a new confocal scanning laser ophthalmoscope]. Ophthalmologe 1997; 94(6): 385-391.

19 Ahmed SS, Lott MN, Marcus DM. The macular xanthophylls. Surv Ophthalmol 2005; 50(2): 183-193.

20 Rothenbuehler SP, Wolf-Schnurrbusch UE, Wolf S. Macular pigment density at the site of altered fundus autofluorescence. Graefes Arch Clin Exp Ophthalmol 2011; 249(4): 499-504.

21 Wüstemeyer H, Jahn C, Nestler A, Barth T, Wolf S. A new instrument for the quantification of macular pigment density: first results in patients with AMD and healthy subjects. Graefes Arch Clin Exp Ophthalmol 2002; 240(8): 666-671.

22 Trieschmann M, Heimes B, Hense HW, Pauleikhoff D. Macular pigment optical density measurement in autofluorescence imaging: comparison of one- and twowavelength methods. Graefes Arch Clin Exp Ophthalmol 2006; 244(12): 1565-1574.

23 Charbel Issa P, van der Veen RLP, Stijfs A, Holz FG, Scholl HP, Berendschot TT. Quantification of reduced macular pigment optical density in the central retina in macular telangiectasia type 2. Exp Eye Res 2009; 89(1): 25-31.

24 Lima VC, Rosen RB, Maia M, Prata TS, Dorairaj S, Farah ME et al. Macular pigment optical density measured by dualwavelength autofluorescence imaging in diabetic and nondiabetic patients: a comparative study. Invest Ophthalmol Vis Sci 2010; 51(11): 5840-5845.

25 Weigert G, Kaya S, Pemp B, Sacu S, Lasta M, Werkmeister $\mathrm{RM}$ et al. Effects of lutein supplementation on macular pigment optical density and visual acuity in patients with age-related macular degeneration. Invest Ophthalmol Vis Sci 2011; 52(11): 8174-8178.

26 Stringham JM, Garcia PV, Smith PA, McLin LN, Foutch BK. Macular Pigment and visual performance in glare: benefits for photostress recovery, disability glare and visual discomfort. Invest Ophthalmol Vis Sci 2011; 52(10): 7406-7415.

27 Sasamoto Y, Gomi F, Sawa M, Tsujikawa M, Nishida K. Effect of 1-year lutein supplementation on macular pigment optical density and visual function. Graefes Arch Clin Exp Ophthalmol 2011; 249(12): 1847-1854.

28 Helb HM, Charbel Issa P, VDV RL, Berendschot TT, Scholl HP, Holz FG. Abnormal macular pigment distribution in type 2 idiopathic macular telangiectasia. Retina 2008; 28(6): 808-816.

29 Davies NP, Morland AB. Color Matching in Diabetes: Optical Density of the Crystalline Lens and Macular Pigments. Invest Ophthalmol Vis Sci 2002; 43(1): 281-289.

30 Sasamoto Y, Gomi F, Sawa M, Sakaguchi H, Tsujikawa M, Nishida K. Effect of cataract in evaluation of macular pigment optical density by autofluorescence spectrometry. Invest Ophthalmol Vis Sci 2011; 52(2): 927-932. 